\title{
Multiple attenuation techniques on OBC survey in Campos basin
}

Pedro Barros*, Marcela Ortin, Jodi Gaeman, Joshua Tunninclife, Frederico Xavier de Melo, Schlumberger; Marcella Rapini, Antonio Jorge Sá, Jorge Nicolas Hounie João Logrado, Petrobras.

Copyright 2019, SBGf - Sociedade Brasileira de Geofísica

This paper was prepared for presentation during the $16^{\text {th }}$ International Congress of the Brazilian Geophysical Society held in Rio de Janeiro, Brazil, 19-22 August 2019.

Contents of this paper were reviewed by the Technical Committee of the $16^{\text {th }}$ International Congress of the Brazilian Geophysical Society and do not necessarily represent any position of the SBGf, its officers or members. Electronic reproduction or storage of any part of this paper for commercial purposes without the written consent of the Brazilian Geophysical Society is prohibited.

\section{Abstract}

Multiple attenuation is one of the biggest challenges in the seismic signal processing workflow in Campos basin. This comes from the seafloor depth variability and multiple reservoir target levels, in both pre-salt and post-salt zones. In this scenario, the main multiples interfere with the primaries, preventing a good reservoir image.

The industry standard approach for multiple prediction is the 3D surface related multiple elimination (SRME) with many good implementations on towed streamer data.

In Ocean Bottom Seismic (OBS) acquisition, sources are near the surface while the receivers are deployed at the seafloor, giving two different datums in the process. This dual datum poses a problem to the use of this technique direct from the OBS data. To overcome this, the traces necessary to the prediction with SRME in the OBS data are obtained from a towed streamer data in the same area.

Another effective demultiple technique is the one based on the source-side wavefield propagation of the water layer to model the related multiples as described by Kostov et al. (2015) with the Generalized Deterministic Water bottom Demultiple (GDWD).

Here we show a case study in Campos Basin, offshore Brazil, where towed streamer complements ocean bottom cable $(\mathrm{OBC})$ data to generate a 3D SRME multiple model and then combined with water-layer wavefield propagation model to produce an improved multiple attenuation from shallow to deep water. We look at the models and its adaptive subtraction results.

The streamer-OBC 3D SRME shows a reliable multiple model where the towed streamer data coverage matches the OBC's. Meanwhile, the GDWD produces a complementary model covering most of the previous' weaknesses resulting in a robust combined model.

\section{Introduction}

The reservoir characterization through seismic data in the Campos Basin relies heavily on an adequate multiple attenuation processing. This is due to the varying water bottom depth which creates multiples that interferes with the targets at post- and pre-salt layers.

The multiple prediction based on trace convolution is being widely used as the surface related multiple elimination method (SRME). It was introduced by Veschuur et al. (1992) and was later generalized by Moore and Dragoset (2008) to fit irregular geometries and to include bigger azimuthal coverages. These methods have been standard for towed-streamer projects globally and are based on the principal that one can create a 3D volume for each input trace by the convolution of selected pairs of traces.

Meanwhile, the use of SRME to predict multiples on seabed data by combination with surface data was first showed by Verschuur and Neumann (1999) in a 2D case with well sampled acquisition geometries.

This method was later used in a 3D OBN combined with wide azimuth data on deep water by Zhong et al. (2014) and by Castelan et al. (2016) both in Gulf of Mexico surveys. These examples show the possibility of effectively predicting multiples on ocean-bottom sensor data by combining it with a multiple free towed streamer data that coincides with the area.

Alternatively, methods for water layer multiple suppression by wave-equation modelling have been proposed since Lokshtanov (2001). Moore and Bisley (2006) proposed a flow to model multiples related water-layer and freesurface multiples known as deterministic water-layer demultiple (DWD). This method was later generalized in Kostov et al. (2015) to the flow known as GDWD that can handle a complex topography of the water bottom multiple generators. This method has been successful on ocean bottom seismic as shown by Ortin et al. (2018).

In this presentation, a reprocessing of an $\mathrm{OBC}$ acquisition in Campos basin is presented. This data covers a shallow to deep water region covered by an orthogonal geometry OBC survey (Vieira et al. 2011) and a narrow azimuth towed streamer data. We look at the process developed for the multiple attenuation on this project, the multiple model predicted and some considerations on the process.

\section{Method}

The 3D SRME algorithm assumes that a free surface multiple can be described as the convolution of two traces. Therefore, the prediction of the multiple on a target trace can be done by convolving two traces, one trace from the source to the reflection point at surface and the another from a source at the same point the receiver. By combining pairs of traces around the target trace a $3 D$ volume is created that is the multiple contribution gather (MCG). The stacking of this volume produces the predicted free-surface multiples. Figure 1Error! Reference source not found. is an illustration of the multiple prediction on a streamer data.

In the OBC case, the symmetry between the two sides from the surface reflection point seen on the towed streamer case described before does not exist. Ikele (1999) describes the surface multiple as a combination of the 
primary events of a streamer and an OBC data by the equation

$D_{1}\left(x_{s}, \xi_{r}, \omega\right)=\int_{-\infty}^{\infty} d x{E^{\prime}}_{0}\left(x_{s}, x, \omega\right) D_{0}\left(x, \xi_{r}, \omega\right)$

where, $D_{1}\left(x_{s}, \xi_{r}, \omega\right)$ is the predicted multiple at the $1^{\text {st }}$ order at the source point at surface $x_{s}$ and receiver point at sea bed $\xi_{\mathrm{r}}, \mathrm{E}_{\mathrm{o}}\left(\mathrm{x}_{\mathrm{s}}, \xi, \omega\right)$ streamer data and $\mathrm{D}_{0}\left(\mathrm{x}, \xi_{\mathrm{r}}, \omega\right)$ the OBC data. The Figure 2 is the illustration of this process on a downgoing wavefield, where the streamer data is used in place of the source side trace for the convolution while the downgoing OBC data is used for the receiver side.

For the described prediction, it can be implied that the streamer to OBC match in terms of temporal sampling, wavelet signature (i.e. receiver ghost removal on the streamer data) and spatial sampling can improve the model results. However, the spatial sampling can be less rigid using the GSMP trace searching algorithm (Moore and Dragoset, 2008).

The preconditioning of the data should be different for each dataset. The sea bed data need to be sorted into common receiver. In this case the searching algorithm should not allow reciprocity between shot and receiver locations. The towed streamer data is sorted into common shot gathers. The reciprocity for this data can be used and the search radius should be large enough to accommodate the azimuth and sampling differences to the OBC.

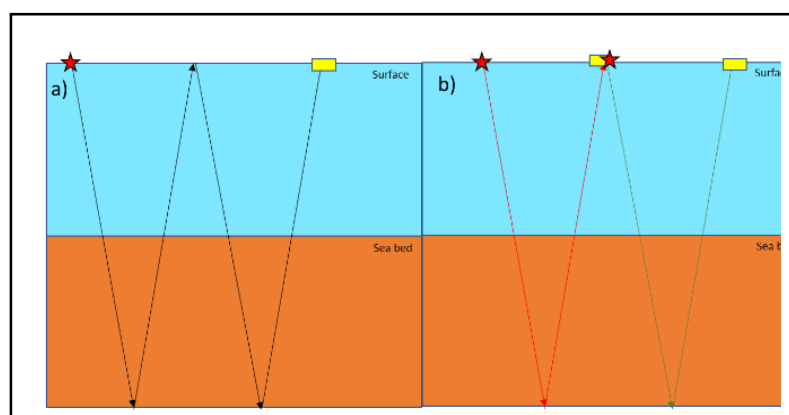

Figure 1: Towed streamer case. Stars show source locations and rectangles are receivers. a) raypath of a $1^{\text {st }}$ order multiple of a target trace. b) traces convolved for prediction, where the red is the sourcereflection and the green is the reflection-receiver.

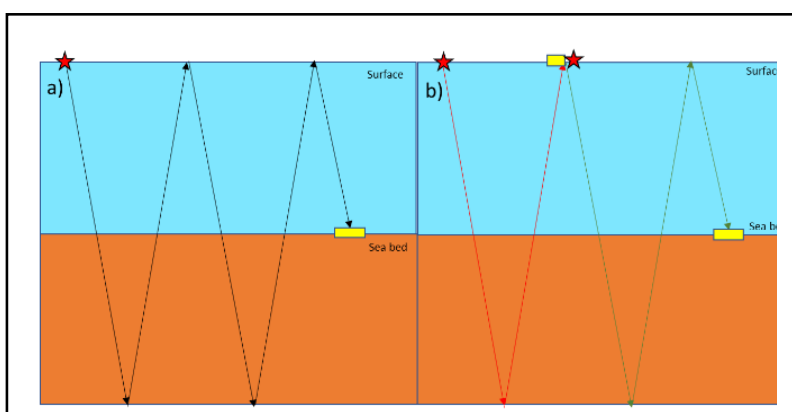

Figure 2: OBC case. Stars show source locations and rectangles are receivers. a) raypath of a $1^{\text {st }}$ order multiple of a target trace on downgoing field. b) traces convolved for prediction, where the red is the source-reflection from the streamer data and the green is the reflection-receiver from the OBC data.
As a complementary method for the prediction of the multiples, a model driven method can be used to provide a more reliable outcome in shallow water areas. Moore and Bisley (2006) described the DWD method that consists in computing a model for water layer multiples by computing a Green's Function of the seafloor reflections based on a water column model. Kostov et al. (2015) extended this scope by introducing GDWD algorithm, that includes mutlidimensional convolutions from the 3D SRME method between the recorded data with a dense pre-defined spatial distribution of traces corresponding to the seafloor Green's Function. These convolutions are again more efficiently done by the special implementation of 3D SRME developed by Moore and Dragoset (2008) as described previously.

\section{Case study}

The studied OBC data was acquired in two parts during 2010-2012 and 2013-2014. Most of the project is in deep water, with a smaller portion on shelf and shallow water. It was shot in with an orthogonal geometry. The receiver cables used were $6 \mathrm{~km}$ long spaced by $300 \mathrm{~m}$ and laid by patches. The shot lines were also spaced by $300 \mathrm{~m}$ with $37.5 \mathrm{~m}$ shot spacing. This configuration resulted in a nominal maximum offset of $6 \mathrm{~km}$ in the primary direction and $3 \mathrm{~km}$ in the secondary.

The survey covers various geological targets with producing post-salt fields that have been in production for many years and more recently discovered pre-salt reservoirs. The reprocessing of these data had the objective of improving the post-salt definition and achieve good structural pre-salt image that was not reached in the previous processing.

The goal is to improve the targets imaging through removing the source-side ghost to generate a broadband seismic data, improve the multiple attenuation and generate a better earth model and using optimal migration algorithms. For demultiple part, the use of 3D SRME prediction with the towed streamer data and wave-field propagation for water layer multiples combined revealed to be the most suitable solution to be applied.

Before the multiple attenuation, the hydrophone and vertical geophone acquired data were combined into upand downgoing wavefields. These then went through the process of source ghost removal with adaptive deghosting (Rickett, 2014) and a water velocity correction to compensate for the variation of seasonal temperature and salinity variations during the campaigns.

The auxiliary towed streamer data was acquired in 2011 and processed previously up to the demultiple phase. It was a narrow azimuth survey in line with the main $O B C$ direction. Its acquisition was done using WesternGeco's QMarine technology with $6 \mathrm{Km}$ cables. Therefore, it was possible to cover most of the input data, however missing the quality at the secondary direction longer offsets. The Figure 3 shows how the OBC and towed streamer surveys overlay each other.

With the objective to improve the model quality a matching filter was designed to make both surveys similar in frequency and phase contents. The resulting multiple 
model will then have the combined frequency bandwidth which, in this case, does not contain the higher frequency from the $\mathrm{OBC}$ as the streamer data has a bigger temporal sampling rate ( $4 \mathrm{~ms}$ opposed to the $3 \mathrm{~ms}$ of the $\mathrm{OBC}$ ).

To handle the areas with lack of coverage, azimuthal variety and the lower frequency bandwidth from the streamer data the GDWD algorithm was used. Water layer Green's Functions were computed using the nominal geometry of the OBC acquisition, with sources and receivers placed at the sea surface level, which is then used in the source side of the SRME to predict another multiple model.

After the predictions, each model has different characteristics, being the GDWD model more comprehensive both spatially and due to its frequency content and the 3D SRME more capable of modeling more complex multiples. Therefore, to achieve the best subtraction of the multiples from the data least-squares adaptive filters were used. First each model is match to the input data separately then both simultaneously. With this, it is expected that not only the GDWD works where the towed streamer is lacking coverage but also it can enhance the overall subtraction by keeping the best events from each method.

This flow was used for both up and downgoing wavefields, although all the examples presented here are from the downgoing wavefield. Required 3D SRME aperture was used by iterative testing to find the best balance between modelling of multiple diffractions or complex areas and avoid the introduction of noises and improve the efficiency of the flow.



Figure 3: Map survey positions where: red polygon represents the limits of the OBC sources; Green dots are the source locations of the towed streamer survey.

\section{Results}

An example of the modelling results can be seen on Figure 4Error! Reference source not found.. Most of the multiples were predicted by the methods and matches with the observed data. By these images, it can be noted that the models have different characteristics most notably on the frequency content and number of events predicted. General differences between the models and the input were well adjusted by the adaptive filters applied producing a more precise combined model.

TheError! Reference source not found. Figure 5Error! Reference source not found. shows the frequency spectra of the different results shown on the previous figure. The method has been able to balance the spectrum on the frequencies up to $80 \mathrm{~Hz}$; the 3D SRME multiple model has a maximum frequency of $100 \mathrm{~Hz}$ due to the towed-streamer data sampling; the GDWD model has a complete frequency content as seen in the input.

In the stack examples, Figure 6,Error! Reference source not found. it can be noticed the multiples has been well attenuated by the combination of the models. By comparing the 3D SRME versus GDWD model, generally, the first one is better at predicting some diffractions multiples while the latter has a frequency content closer to the input data. The combination of the models shows that the adaptive filtering keeps the main predicted events from each method.

\section{Conclusions}

The described 3D SRME method has the advantage of being data driven and has a good flexibility over a survey with varying depth water bottom. However, this flow is limited by the sampling and general data quality of both surveys. Meanwhile, the GDWD model shows a better result modelling the main multiple events with a more suited frequency content,overcoming the lack of sampling however its designed to only target water-layer multiples.

In conclusion, the combination of the 3D SRME flow using towed streamer and $\mathrm{OBC}$ data as data-driven model with a wavefield propagated model has been shown viable to the Campos basin enviroment with ocean bottom sensor acquisition in cross-spread geometries and has demonstrated satisfactory result in multiple attenuation

\section{Acknowledgments}

We thank Schlumberger and Petrobras for the permission to present this work. We also acknowledge Matthew Salgadoe and Abhishek Raj for their contributions during this work.

\section{References}

VERSCHUUR, D. J., BERKHOUT, A. J. and WAPENAAR, C. P. A. Adaptive surface-related multiple elimination. Geophysics, V. 57, NO. 9, p.1166-1177, 1992.

DRAGOSET, B. and MOORE, I. General surface multiple prediction: A flexible 3D SRME algorithm. First Break, 26, September, 89-100, 2008.

VERSCHUUR, D.J. and NEUMANN, E.I. Integration of OBS data and surface data for OBS multiple removal. 69th Annual International Meeting, SEG, Expanded Abstracts, 1350-1353, 1999.

ZHONG, R., CHAO, J.M, SHUO, J. and XUAN, Y. U Incorporating streamer data for OBN free-surface multiple prediction - a case study in deepwater Gulf of Mexico. SEG Expanded Abstracts, 4108-4112, 2014.

CASTELAN, A. R., KOSTOV, C., SARAGOUSSI, E., DE MELO, F. X., MIERS, G., WU, Z., ABDELAZIZ, K. 
MATARACIOGLU, O., KRISTIANSEN, P., SLATON, S., CHAKRABORTY, S. and LI, Q. OBN multiple attenuation using OBN and towed-streamer data: Deepwater Gulf of Mexico case study, Thunder Horse Field, SEG Expanded Abstracts, 4513-4517, 2016.

LOKSHTANOV, D. Suppression of water-layer multiples and peg-legs by wave-equation approach, 63rd Conference and Exhibition, EAGE, Extended Abstracts, IM-1, 2001.

MOORE, I., and BISLEY R. Multiple attenuation in shallow water situations, 68th Conference and Exhibition, EAGE, Extended Abstracts, F018, 2006.

KOSTOV, C., DE MELO, F.X., RAJ, A., ZARKHIDZE, A., COOKE, A., MIERS, G. \& BACON, J. Multiple attenuation for shallow-water surveys: Notes on old challenges and new opportunities, Leading Edge, vol. 34, no. 7, pp. 760768, 2015.

ORTIN, M., SALGADOE, M., FENOGLIO, F., RAJ, A., SANCHEZ, M., DERHAROUTIAN, D., VAZQUES, A., DOMINGUEZ, S., DE LEON, J. D. Large-scale, shallowwater, ocean-bottom seismic project in southern Gulf of Mexico, 2018 SEG International Exposition and Annual Meeting, SEG 2018, pp. 3759, 2018

VIEIRA, J. M. B., CHAVES, B., FORMIGA, L., LIMA, A.P. and MATHIESON, A. Full azimuth OBC acquisition - A Campos Basin pre-salt case study, XII International Congress of the Brazilian Geophysical Society, Rio de Janeiro, 2011.

IKELLE, L. T. Combining two seismic experiments to attenuate free-surface multiples in OBC data, Geophysical Prospecting, vol. 47, pp. 179-193, 1999

RICKETT, J.E., VAN MANEN, D.J., LOGANATHAN, P. and SEYMOUR, N. Slanted-streamer Data-adaptive Deghosting with Local Plane Waves, 76th European Association of Geoscientists and Engineers Conference and Exhibition 2014: Experience the Energy - Incorporating SPE EUROPEC 2014, pp. 572. 

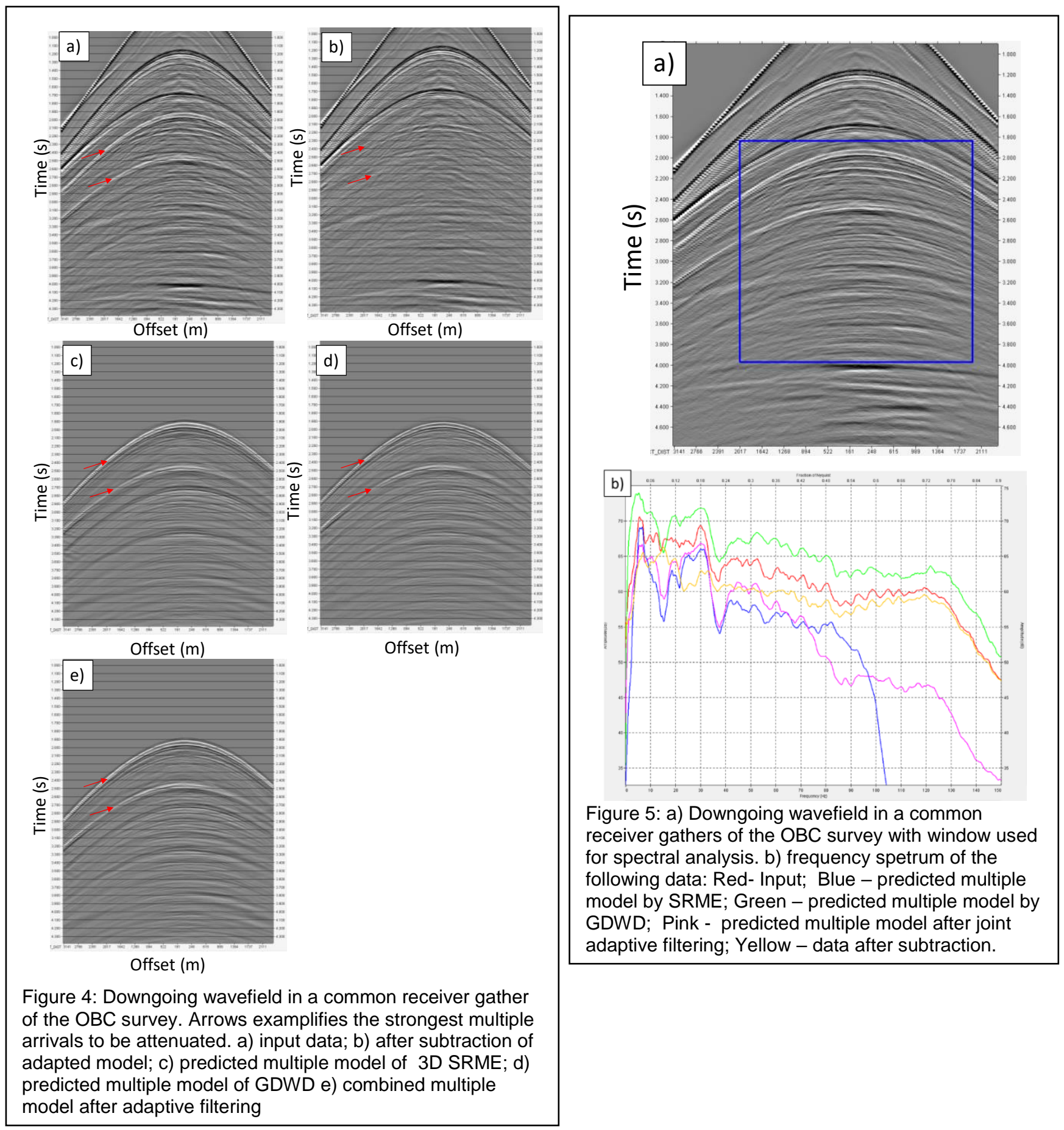


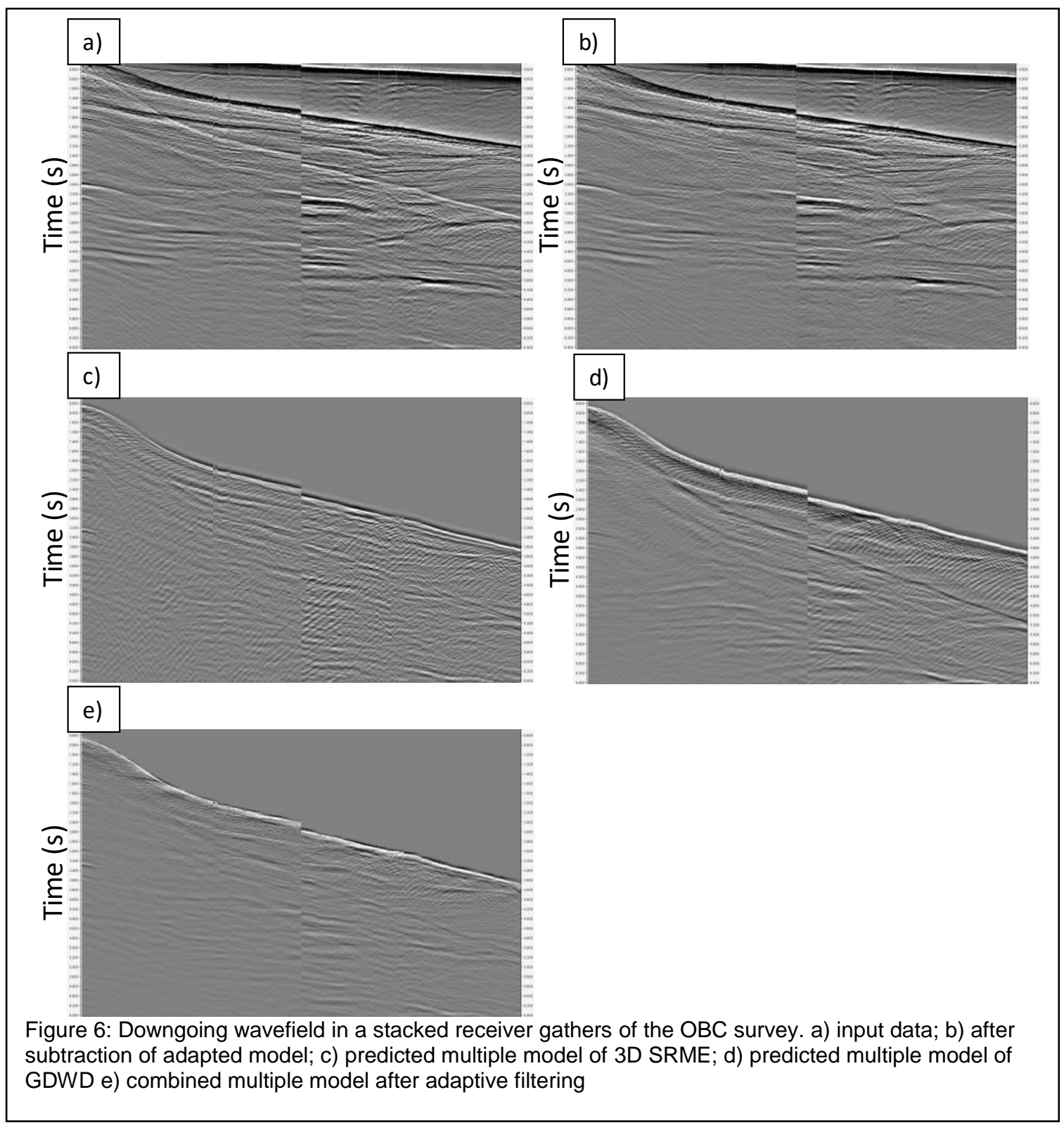

